PROCEEDINGS OF THE

AMERICAN MATHEMATICAL SOCIETY

Volume 140, Number 2, February 2012, Pages 677-689

S 0002-9939(2011)10926-2

Article electronically published on June 9, 2011

\title{
LIPSCHITZ CLASS, NARROW CLASS, AND COUNTING LATTICE POINTS
}

\author{
MARTIN WIDMER
}

(Communicated by Matthew A. Papanikolas)

\begin{abstract}
A well-known principle says that the number of lattice points in a bounded subset $S$ of Euclidean space is about the ratio of the volume and the lattice determinant, subject to some relatively mild conditions on $S$. In the literature one finds two different types of such conditions: one asserts the Lipschitz parameterizability of the boundary $\partial S$, and the other one is based on intersection properties of lines with $S$ and its projections to linear subspaces. We compare these conditions and address a question, which we answer in some special cases. Then we give some simple upper bounds on the number of lattice points in a convex set, and finally, we apply these results to obtain estimates for the number of rational points of bounded height on certain projective varieties.
\end{abstract}

\section{INTRODUCTION}

The counting of lattice points in a given bounded subset of the Euclidean space $\mathbb{R}^{n}$ is an important issue in many parts of number theory and other branches of mathematics. If the set, say $S$, behaves nicely, then the ratio $\operatorname{Vol}(S) / \operatorname{det} \Lambda$ of the volume and the lattice determinant is a good estimate for the cardinality $|S \cap \Lambda|$. In the literature there are two different approaches to formally define the term "nicely". The older one is associated with the name of Lipschitz. Roughly speaking it says that the boundary $\partial S$ of $S$ can be parameterized by a Lipschitz map with a reasonably small Lipschitz constant. The second approach goes back to Davenport and was further developed by Schmidt. Here one has to control the diameter of $S$ and the number of connected components of the intersection of each line with $S$ and with all of its projections on linear subspaces. Both conditions yield similar counting results, and it is therefore natural to ask how they are related. Masser and Vaaler 9] pointed out that the Lipschitz condition certainly does not imply the Davenport condition but that the other implication possibly holds in some form. One aim of this note is to render this "question" precise and to answer it in the case where $S$ is convex or when $n=2$ and some extra conditions hold. The proof of the first assertion rests on John's Theorem for convex sets and simple planimetric arguments; the proof of the second assertion relies on results from integral geometry such as Poincaré's formula.

Masser and Vaaler [9] and the author [15, 13] have introduced Lipschitz heights and adelic Lipschitz heights and proved general counting results regarding these.

Received by the editors August 3, 2010 and, in revised form, November 23, 2010.

2010 Mathematics Subject Classification. Primary 52A30, 11H06; Secondary 11P21, 11D45.

Key words and phrases. Lattice points, counting, Lipschitz class, narrow class, height.

(C)2011 American Mathematical Society 677 
This led to asymptotic estimates for the number of: algebraic points of fixed degree and bounded Weil height in projective space [9, [13] and on linear varieties [14, algebraic numbers of bounded Weil height satisfying certain subfield conditions [16, and connected algebraic subgroups of the torus of bounded degree [9]. It is likely that further applications will follow. But to apply the general counting results from [9, [15, 13] one needs to verify that certain balls, associated to the distance functions of the heights, have Lipschitz parameterizable boundaries. In many applications these balls are convex. Therefore it is convenient to have a general result, such as Theorem 2.6, which proves the required assertion of Lipschitz parameterizability in all of these cases.

Moreover, we prove an estimate for $|S \cap \Lambda|$ when $S$ is convex. Estimates of this type are well known and follow immediately from a result of Blichfeldt, but we will give another proof, again relying on John's Theorem. Finally, we illustrate the use of such estimates by an example on counting rational points on certain algebraic varieties.

\section{Definitions AND RESUlts}

Throughout this article, $n$ denotes an integer with $n \geq 2, M$ and $s$ will always stand for a positive integer, while $L$ will denote a nonnegative real number. By a lattice in $\mathbb{R}^{n}$ we mean the $\mathbb{Z}$-span of $n$ linearly independent vectors $v_{1}, \ldots, v_{n}$ in $\mathbb{R}^{n}$. The determinant of the lattice is then given by the modulus of the determinant of the matrix whose columns are $v_{1}, \ldots, v_{n}$. For a vector $\mathbf{x}$ in $\mathbb{R}^{n}$ we write $|\mathbf{x}|$ for the Euclidean length of $\mathbf{x}$. We will use vector and point synonymously. For a point $P$ in $\mathbb{R}^{n}$ and a real $R>0$ we write $B_{P}(R)$ for the closed Euclidean ball with radius $R$ centered at $P$. The successive minima $\lambda_{1}, \ldots, \lambda_{n}$ of a lattice $\Lambda$ in $\mathbb{R}^{n}$ are understood in Minkowski's sense with respect to the unit ball $B_{0}(1)$; i.e., for $i=1, \ldots, n$,

$$
\lambda_{i}=\inf \left\{\lambda: B_{0}(\lambda) \cap \Lambda \text { contains } i \text { linearly independent vectors }\right\} .
$$

The following definition has its origin in a paper of Davenport 4, in which a counting theorem for lattice points of $\mathbb{Z}^{n}$ was proved. Later Schmidt [12, p. 347] adjusted the definition to handle more general lattices, and finally Gao [5, p. 14] slightly refined Schmidt's definition, replacing "compact" by "bounded and measurable". The definition in [5] is as follows.

Definition 2.1. A subset $S$ of $\mathbb{R}^{n}$ is said to be of narrow class $s$ if

(a) $S$ is bounded, measurable and intersects every line in at most $s$ intervals or single points.

(b) The same is true for any projection of $S$ on any linear subspace of $\mathbb{R}^{n}$.

Note that a set of narrow class 1 is simply a bounded convex set and that a connected subset of $\mathbb{R}^{2}$ is of narrow class $s$ if it satisfies condition $(a)$. The Lipschitz approach requires the following condition.

Definition 2.2. We say that a set $S$ is in $\operatorname{Lip}(n, M, L)$ (or of Lipschitz class $(n, M, L))$ if $S$ is a subset of $\mathbb{R}^{n}$, and if there are $M$ maps $\phi_{1}, \ldots, \phi_{M}:[0,1]^{n-1} \longrightarrow$ $\mathbb{R}^{n}$ satisfying a Lipschitz condition

$$
\left|\phi_{i}(\mathbf{x})-\phi_{i}(\mathbf{y})\right| \leq L|\mathbf{x}-\mathbf{y}| \text { for } \mathbf{x}, \mathbf{y} \in[0,1]^{n-1}, i=1, \ldots, M,
$$

such that $S$ is covered by the images of the maps $\phi_{i}$. 
We call $L$ a Lipschitz constant for the maps $\phi_{i}$. The following theorem was proved by Schmidt, first in a simpler version ([12, Lemma 1) and then (see [5], p. 15) in the stated form.

Theorem 2.3 (Schmidt). Let $\Lambda$ be a lattice in $\mathbb{R}^{n}$ with successive minima $\lambda_{1}, \ldots, \lambda_{n}$. Let $S$ be a set in $\mathbb{R}^{n}$ of narrow class $s$ and assume $S \subseteq B_{0}(R)$. Then we have

$$
|| S \cap \Lambda\left|-\frac{\operatorname{Vol}(S)}{\operatorname{det} \Lambda}\right| \leq c_{1}(n, s) \max _{0 \leq i<n} \frac{R^{i}}{\lambda_{1} \cdots \lambda_{i}} .
$$

For $i=0$ the expression in the maximum is to be understood as 1 . Moreover, one can choose $c_{1}(n, s)=\left(s+\frac{2}{\sqrt{3}} 3^{n} n\right)^{n}$.

Unfortunately, the author is not aware of any published reference for Theorem 2.3. Therefore we will not make use of it. However, an analogous result was obtained in [15] (Theorem 5.4) when the boundary of the set lies in $\operatorname{Lip}(n, M, L)$.

Theorem 2.4. Let $\Lambda$ be a lattice in $\mathbb{R}^{n}$ with successive minima $\lambda_{1}, \ldots, \lambda_{n}$. Let $S$ be a bounded set in $\mathbb{R}^{n}$ such that the boundary $\partial S$ of $S$ is in Lip $(n, M, L)$. Then $S$ is measurable, and, moreover,

$$
|| S \cap \Lambda\left|-\frac{\operatorname{Vol}(S)}{\operatorname{det} \Lambda}\right| \leq c_{2}(n) M \max _{0 \leq i<n} \frac{L^{i}}{\lambda_{1} \cdots \lambda_{i}} .
$$

For $i=0$ the expression in the maximum is to be understood as 1 . Furthermore, one can choose $c_{2}(n)=n^{3 n^{2} / 2}$.

It is easy to see that there are sets which are not of narrow class $s$ for any $s$ but which have a boundary of Lipschitz class $(n, M, L)$; e.g., take the square $[0,1 / \pi] \times[0,1 / \pi]$ in $\mathbb{R}^{2}$ where the edge on the $x$-axis is replaced by the curve $\left(x, x^{3} \sin (1 / x)\right)$ for $0<x \leq 1 / \pi$. On the other hand, it has been pointed out in [9, p. 438] that narrow class possibly implies some type of Lipschitz class. We propose the following question.

Question 2.5. Let $S$ be a set in $\mathbb{R}^{n}$ of narrow class $s$, and assume $S$ lies in a ball of radius $R$. Does there exist a natural number $M=M(n, s)$ and a real number $C=C(n, s)$ such that $\partial S$ is in $\operatorname{Lip}(n, M, C R)$ ?

Now suppose $s=1$, which is equivalent to $S$ being convex and bounded. Then it is rather easy to see that $\partial S$ is of Lipschitz class $(n, 1, L)$ for some value of $L$, but it is not so obvious that one can choose $L=C R$ with a constant $C=C(n)$. However, we have the following affirmative answer for Question 2.5.

Theorem 2.6. If $S$ is a set in $\mathbb{R}^{n}$ of narrow class 1 which lies in a ball of radius $R$, then $\partial S$ is in $\operatorname{Lip}\left(n, 1,8 n^{5 / 2} R\right)$.

If $S \subseteq B_{P}(R)$ is of narrow class $s$, then one can try to find convex sets $K_{1}, \ldots, K_{M}$ such that $\partial S$ is covered by the union of the boundaries $\partial K_{i}$. In this case one can apply Theorem [2.6 with the convex sets $B_{P}(R) \cap K_{i}$ to deduce that $\partial S$ lies in $\operatorname{Lip}\left(n, M, 8 n^{5 / 2} R\right)$. Unfortunately $M$ cannot be bounded in terms of $s$ and $n$ only; e.g., consider a ball with many "equidistributed" little spikes on its surface.

For $n=2$ we can apply Poincaré's formula from integral geometry to answer a variation of Question 2.5. In this paper a curve means a continuous map $\varphi$ : $[0,1] \longrightarrow \mathbb{R}^{2}$. We say that the curve $\varphi$ is simple if $\varphi$ is injective on $(0,1)$, closed if $\varphi(0)=\varphi(1)$. Finally, we say that the curve $\varphi=\left(\varphi_{1}, \varphi_{2}\right)$ is piecewise smooth if 
there exist finitely many reals $0=t_{0}<\cdots<t_{N}=1$ such that both components of $\varphi$ have a continuous derivative on $\left(t_{i}, t_{i+1}\right)$ and right-sided (left-sided) derivatives in $t_{i}\left(t_{i+1}\right)$ exist for all $0 \leq i<N$. We call the image $\varphi([0,1])$ the path of the curve $\varphi$. Let $\mathfrak{M}$ be the group of motions $\phi$ in the plane. Any $\phi \in \mathfrak{M}$ has the form

$$
\phi(x, y)=(x \cos \theta-y \sin \theta+a, x \sin \theta+y \cos \theta+b),
$$

where $-\infty<a<\infty,-\infty<b<\infty, 0 \leq \theta \leq 2 \pi$. So each motion is determined by an element $(a, b, \theta)$ of 3 -space. This space, together with the equivalence relation $(a, b, \theta) \sim(a, b, \theta+2 \pi k)(k \in \mathbb{Z})$, is the space of the group of motions, also denoted by $\mathfrak{M}$. The exterior product

$$
\mathrm{d} K=\mathrm{d} a \wedge \mathrm{d} b \wedge \mathrm{d} \theta
$$

is the unique (up to a constant factor) left and right invariant 3 -form on $\mathfrak{M}$ and is called the kinematic density (see [11, p. 85] for a reference).

Definition 2.7. A subset $S$ of $\mathbb{R}^{2}$ is said to be of tight class $s$ if the following two conditions are fulfilled.

(I) The boundary $\partial S$ of $S$ is the path of a simple, closed, piecewise smooth curve.

(II) Let $\Gamma_{0}$ be a line segment. Then the subset $\mathfrak{M}_{0}$ of $\mathfrak{M}$ defined by

$$
\left|\partial S \cap \phi\left(\Gamma_{0}\right)\right|>2 s \text { has measure zero, i.e., } \int_{\mathfrak{M}_{0}} \mathrm{~d} K=0 .
$$

Condition $(I)$ implies that a set $S$ of tight class $s$ is connected, and that $\partial S$ has measure zero. This in turn implies that $S$ is measurable, but unlike a set of narrow class $s$ it may be unbounded. Now suppose that $S$ is of narrow class $s$. A "typical" line $l$ intersects $S$ in $j \leq s$ intervals, whose endpoints are the only boundary points of $S$ on $l$. Of course it can happen that $\partial S \cap l$ contains more than $2 s$ points, but it seems likely that the set $\mathfrak{M}_{l}$ of motions $\phi$ with $|\partial S \cap \phi(l)|>2 s$ has measure zero. We can now state our approach to Question 2.5] in dimension two.

Theorem 2.8. Let $S$ in $\mathbb{R}^{2}$ be a set of tight class $s$, and assume $S$ lies in a ball of radius $R$. Then $\partial S$ is in $\operatorname{Lip}(2,1,2 \pi s R)$.

In fact we will prove that the arc length of $\partial S$ is bounded by $2 \pi s R$. This is best possible, as we can see by the following examples: for $s=1$ we take $S$ as the circle $B_{0}(R)$; for $s$ even we take $S$ as a "worm" coiled $s-1$ times around the slightly smaller circle $B_{0}(R-\epsilon)$; for $s>1$ odd the latter circle should be considered the head of the worm and its tail is coiled $s-2$ times around the head. Note also that the inequality $|\partial S| \leq 2 \pi s R$ generalizes (at least for sets of tight class) the well-known fact that the arc length of the boundary of a convex set in $B_{P}(R) \subseteq \mathbb{R}^{2}$ cannot exceed $\left|\partial B_{P}(R)\right|$. A reference for the latter can be found in [17, p. 15].

Next we aim at giving some upper bounds on the number of lattice points in a subset $S$ of Euclidean space. Let us assume for a moment that $S$ is a bounded convex set in $\mathbb{R}^{n}, \Lambda$ is a lattice in $\mathbb{R}^{n}$, and $S \cap \Lambda$ is not contained in an affine subspace of dimension $n-1$. For $n=2$, Pick's Theorem gives $|S \cap \Lambda| \leq 2 \operatorname{Vol}(S) / \operatorname{det} \Lambda+2$. From an article of Henk and Wills [6] the author has learned that Blichfeldt [2] generalized this to arbitrary dimensions $n$, i.e., $|S \cap \Lambda| \leq n ! \operatorname{Vol}(S) / \operatorname{det} \Lambda+n$. Unfortunately the author was unable to get a copy of Blichfeldt's article [2]. Henk and Wills [6] have shown $|S \cap \Lambda| \leq \operatorname{Vol}(S) / \operatorname{det} \Lambda+c n(n-1) ! F(S) / \operatorname{det} \Lambda_{n-1}$, where $c$ 
is a universal constant, $F(S)$ denotes the surface area, and $\operatorname{det} \Lambda_{n-1}$ is the minimal determinant of an $(n-1)$-dimensional sublattice of $\Lambda$. For our applications the concrete dependence on $n$ is not important, but we have to sum these estimates over an infinite set of lattices, and thus the " $+n$ " and even " $+F(S) / \operatorname{det} \Lambda_{n-1}$ " may cause problems. However, in dimension 2 a simple triangulation argument shows that $|S \cap \Lambda| \leq 6 \operatorname{Vol}(S) / \operatorname{det} S$. A higher-dimensional version follows easily from Blichfeldt's result: $S \cap \Lambda$ is not contained in an affine subspace of dimension $n-1$ means we can find $w, v_{1}, \ldots, v_{n}$ in $S \cap \Lambda$, such that $v_{1}-w, \ldots, v_{n}-w$ are linearly independent. Now the convex hull of $w, v_{1}, \ldots, v_{n}$ lies in $S$ and has volume at least $\operatorname{det} \Lambda / n$ !. Thus $|S \cap \Lambda| \leq(n+1) ! \operatorname{Vol}(S) / \operatorname{det} \Lambda$. We will give another proof, relying on John's Theorem, but first we introduce some more notation. For a nonempty subset $H$ of $\mathbb{R}^{n}$ we define $\mathcal{A}_{H}$ to be the minimal affine subspace containing $H$. Note that $\operatorname{dim} \mathcal{A}_{H}=0$ if and only if $|H|=1$. For $1 \leq j \leq n$ let us write $\operatorname{Vol}_{j}$ for the $j$-dimensional Lebesque measure. If $S$ is a bounded convex set in $\mathbb{R}^{n}$, then $\operatorname{Vol}_{j}(S \cap \mathcal{A})$ is well defined for any affine subspace $\mathcal{A}$ of dimension $j$.

Proposition 2.9. Let $\Lambda$ be a lattice in $\mathbb{R}^{n}$ with successive minima $\lambda_{1}, \ldots, \lambda_{n}$. Let $S$ be a bounded convex set in $\mathbb{R}^{n}$. Suppose $|S \cap \Lambda|>1$ and set $l=\operatorname{dim} \mathcal{A}_{S \cap \Lambda}$. Then we have

$$
|S \cap \Lambda| \leq c_{3}(l) \frac{\operatorname{Vol}_{l}\left(S \cap \mathcal{A}_{S \cap \Lambda}\right)}{\lambda_{1} \cdots \lambda_{l}} .
$$

One can choose $c_{3}(l)=8^{l} l^{3 l(l / 2+1)}$.

Now suppose $|S \cap \Lambda|$ is not contained in an $(n-1)$-dimensional affine subspace. This means that $\operatorname{dim} \mathcal{A}_{S \cap \Lambda}=n$, and thus we deduce, using Minkowski's Second Theorem, the following corollary.

Corollary 2.10. Let $\Lambda$ be a lattice in $\mathbb{R}^{n}$ and let $S$ be a bounded convex set in $\mathbb{R}^{n}$. Suppose $S \cap \Lambda$ is not contained in an affine subspace of dimension $n-1$. Then we have

$$
|S \cap \Lambda| \leq c_{4}(n) \frac{\operatorname{Vol}(S)}{\operatorname{det} \Lambda} .
$$

One can choose $c_{4}(n)=\frac{\pi^{n / 2} n !}{2^{n} \Gamma(n / 2+1)} c_{3}(n)$.

Now let $d$ be a fixed positive integer. We apply the latter result to deduce an estimate for the number of rational points of bounded height on the algebraic variety $V_{d} \subseteq \mathbb{P}^{2} \times \mathbb{P}^{2}$ defined by

$$
x_{0} y_{0}^{d}+x_{1} y_{1}^{d}+x_{2} y_{2}^{d}=0 .
$$

Let $H_{n}$ be the multiplicative Weil height on $\mathbb{P}^{n}(\mathbb{Q})$; so if $P=\left(x_{0}: \ldots: x_{n}\right)$ with coprime integers $x_{0}, \ldots, x_{n}$, then $H_{n}(P)=\max \left\{\left|x_{0}\right|, \ldots,\left|x_{n}\right|\right\}$. We define the height $H$ on $\mathbb{P}^{2}(\mathbb{Q}) \times \mathbb{P}^{2}(\mathbb{Q})$ via the Segre embedding $\sigma: \mathbb{P}^{2} \times \mathbb{P}^{2} \rightarrow \mathbb{P}^{8}$ as $H(P, Q)=$ $H_{8}(\sigma(P, Q))$. Then we have $H(P, Q)=H_{2}(P) H_{2}(Q)$. Let $N\left(V_{d}, t\right)$ be the counting function

$$
N\left(V_{d}, t\right)=\left|\left\{(P, Q) \in V_{d}(\mathbb{Q}): H(P, Q) \leq t\right\}\right| .
$$


Corollary 2.11. For $t \geq 1$ and any $\epsilon>0$ we have

$$
t^{2} \ll N\left(V_{d}, t\right) \ll_{\epsilon} \begin{cases}t^{9 / 4}: & \text { if } d=1, \\ t^{2+\epsilon}: & \text { if } d=2, \\ t^{2}: & \text { if } d \geq 3 .\end{cases}
$$

The implicit constant in " $\ll_{\epsilon}$ " depends on $\epsilon$ if $d=2$ and only on $d$ if $d \neq 2$.

Of course the lower bound is trivial; just fix e.g. $\left(y_{0}: y_{1}: y_{2}\right)=(1: 0: 0)$ and count the points $\left(0: x_{1}: x_{2}\right)$. The upper bound $\ll t^{9 / 4}$ can easily be shown using only Corollary 2.10, But for $d>1$ we also need a general result of Pila.

\section{Proof of Theorem 2.6}

The proof of Theorem 2.6 rests on John's Theorem and the following lemma.

Lemma 3.1. Let $S$ in $\mathbb{R}^{n}$ be a convex set, $P$ a point in $S$ and $r, R$ positive reals such that

$$
B_{P}(r) \subseteq S \subseteq B_{P}(R) .
$$

Then the boundary $\partial S$ is in $\operatorname{Lip}\left(n, 1,8 \sqrt{n-1} R^{2} / r\right)$.

Proof. Let $P=\left(\zeta_{1}, \ldots, \zeta_{n}\right)$ and let

$$
\varphi:[0,2 \pi] \times[0, \pi]^{n-2} \longrightarrow \partial B_{P}(r)
$$

be the standard parameterization of $\partial B_{P}(r)$ via polar coordinates such that

$$
\begin{array}{lr}
x_{1}=r \cos \theta_{1} \cos \theta_{2} \cos \theta_{3} \cdots \cos \theta_{n-1}+\zeta_{1}, \\
x_{2}=r \sin \theta_{1} \cos \theta_{2} \cos \theta_{3} \cdots \cos \theta_{n-1}+\zeta_{2}, \\
x_{3}=r \sin \theta_{2} \cos \theta_{3} \cdots \cos \theta_{n-1}+\zeta_{3}, \\
\vdots & \\
x_{n}= & r \sin \theta_{n-1}+\zeta_{n} .
\end{array}
$$

Of course for $n=2$ this is to be understood as a map $\varphi:[0,2 \pi] \longrightarrow \partial B_{P}(r)$. Let $A, B$ be different points in $\mathbb{R}^{n}$; then we denote by $[A, B]$ the line segment between $A$ and $B(A, B$ are included). Similarly $(A, B)$ denotes the line segment without the points $A, B$. We claim that intersecting the ray, starting in $P$ and containing $\varphi(\boldsymbol{\theta})$, with $\partial S$, gives a parameterization $\widetilde{\varphi}$ of $\partial S$. It suffices to show that each such ray contains no more than one boundary point of $S$. So assume such a ray contains two distinct boundary points, say $A$ and $B$, where $A$ is closer to $P$. Now consider the union of all line segments $(B, F)$ starting in $B$ and ending on any boundary point $F$ of $B_{P}(r)$. Then each point of the line segment $(B, P)$ lies in the interior of this union. Due to the convexity of $S$ this union of line segments is a subset of the topological closure of $S$, and since $A$ lies in $(B, P)$ we conclude that $A$ lies in the interior of $S$, a contradiction. This argument will be used once more at the end of the proof.

The next step of the proof is to show that $\widetilde{\varphi}$ is a Lipschitz parameterization with Lipschitz constant $(4 / \pi) \sqrt{n-1} R^{2} / r$, so that after normalizing properly to get a map as in (2.1) one gets the Lipschitz constant $8 \sqrt{n-1} R^{2} / r$.

Let us write $\overline{A B}$ for the length of the line segment $[A, B]$. Let $A, B, C$ be three different points. Let $\beta$ be the angle between the line segments $[A, B]$ and $[A, C]$, 
that is, the value in $[0, \pi]$ such that $\cos \beta=\langle B-A, C-A\rangle /(\overline{A B} \cdot \overline{A C})$, where $\langle\cdot, \cdot\rangle$ denotes the Euclidean scalar product. If the points do not lie on one line, then we may consider the triangle $\triangle(A, B, C)$ with vertices $A, B, C$. Let $\widetilde{\varphi}\left(\boldsymbol{\theta}_{\mathbf{1}}\right)=A$, $\widetilde{\varphi}\left(\boldsymbol{\theta}_{\mathbf{2}}\right)=B$ be two distinct points on the boundary of $S$. We may assume

$$
0<\left|\boldsymbol{\theta}_{2}-\boldsymbol{\theta}_{\mathbf{1}}\right|<\frac{\pi}{2 \sqrt{n-1}},
$$

for otherwise we get automatically

$$
\overline{A B} \leq 2 R \leq(4 / \pi) \sqrt{n-1} R\left|\boldsymbol{\theta}_{\mathbf{2}}-\boldsymbol{\theta}_{\mathbf{1}}\right| .
$$

Denote by $\alpha$ the angle between $[P, A]$ and $[P, B]$, and write $|\cdot|_{l_{1}}$ for the $l_{1}$-norm. Then we have

$$
\alpha \leq\left|\boldsymbol{\theta}_{\mathbf{2}}-\boldsymbol{\theta}_{\mathbf{1}}\right|_{l_{1}}
$$

which is a simple consequence of the triangle inequality in the metric space $\mathbb{S}^{n-1}$ (see p. 17 in [3]). Hence

$$
\alpha \leq \sqrt{n-1}\left|\boldsymbol{\theta}_{\mathbf{2}}-\boldsymbol{\theta}_{\mathbf{1}}\right|
$$

If $A, B, P$ lie on a common line, then either $\alpha=\pi$ and so $\left|\boldsymbol{\theta}_{\mathbf{2}}-\boldsymbol{\theta}_{\mathbf{1}}\right| \geq \pi / \sqrt{n-1}$ or $A=B$, both contradicting our assumptions. So the lines joining $P, A$ and $P, B$ span a plane, say $\mathcal{P}$. Write $\mathcal{B}$ for the interior of $B_{P}(r)$ and $\mathcal{L}$ for the line joining $A$ and $B$. The line in $\mathcal{P}$ perpendicular to $\mathcal{L}$, which joins $P$, intersects $\mathcal{L}$ in a point denoted by $C$. The proof splits into the following three cases:

(1) $\mathcal{L}$ does not meet $\mathcal{B}(C$ not in $\mathcal{B})$.

(2) $\mathcal{L}$ meets $\mathcal{B}$ between $A$ and $B(C$ is in $[A, B]$ and in $\mathcal{B})$.

(3) The remaining case $(A$ is in $[B, C]$ or $B$ is in $[A, C]$, and $C$ is in $\mathcal{B})$.

We start with the first case. Now $\mathcal{L}$ does not meet $\mathcal{B}$ is equivalent to $\overline{P C} \geq r$. The area of $\triangle(P, A, B)$ is $\overline{P C} \cdot \overline{A B} / 2$. It is clear that $\triangle(P, A, B)$ does not exceed the area of a sector of $B_{P}(R) \cap \mathcal{P}$ with angle $\alpha$, which is $\alpha R^{2} / 2$. Thus

$$
\overline{A B} \leq \frac{R^{2}}{r} \alpha .
$$

For the second case we have that $[A, B]$ contains $C$. Denote the angle between $[P, C]$ and $[P, A]$ by $\alpha_{1}$, and the angle between $[P, C]$ and $[P, B]$ by $\alpha_{2}$, so that $\alpha=\alpha_{1}+\alpha_{2}$ and $0 \leq \alpha_{1}, \alpha_{2} \leq \alpha \leq \pi / 2$. Hence

$$
\overline{A C}=\overline{P A} \sin \alpha_{1} \leq \overline{P A} \alpha_{1} \leq R \alpha_{1},
$$

and similarly $\overline{B C} \leq R \alpha_{2}$, leading to

$$
\overline{A B} \leq R \alpha .
$$

Since $A, B$ lie on the boundary of $S$, none of them can lie in $\mathcal{B}$. Thus the remaining case occurs if either $A$ is in $[B, C]$ or $B$ lies in $[A, C]$. Since $C$ is in $\mathcal{B}$ there is a positive $\epsilon$ such that $B_{C}(\epsilon)$ lies in $\mathcal{B}$. But now we use the same argument as in the beginning of the proof to show that due to the convexity either $A$ or $B$ lies in the interior of $S$, a contradiction. Thus the remaining case is impossible.

Recalling (3.1) and $R \geq r$ proves that in all cases we have

$$
\left|\widetilde{\varphi}\left(\boldsymbol{\theta}_{\mathbf{1}}\right)-\widetilde{\varphi}\left(\boldsymbol{\theta}_{\mathbf{2}}\right)\right|=\overline{A B} \leq(4 / \pi) \sqrt{n-1} \frac{R^{2}}{r}\left|\boldsymbol{\theta}_{\mathbf{1}}-\boldsymbol{\theta}_{\mathbf{2}}\right| .
$$

Properly normalizing to get a map as in (2.1) gives an additional factor $2 \pi$ and completes the proof. 
We are now in position to prove Theorem 2.6.

Recall that a set of narrow class 1 is convex. Suppose the interior $\operatorname{int}(S)$ of $S$ is empty. Pick $P_{0}$ in $S$; then the points of $S-P_{0}$ cannot span $\mathbb{R}^{n}$ as an $\mathbb{R}$ vector space, else $S$ would contain a small (nonempty) open parallelepiped. Hence $S$ lies in a hyperplane, and so $\partial S$ lies in a ball $B_{P^{\prime}}\left(R^{\prime}\right)$ in $\mathbb{R}^{n-1}$ for some $R^{\prime} \leq R$. So it suffices to know that $B_{P^{\prime}}\left(R^{\prime}\right)$ lies in $\operatorname{Lip}(n, 1,2 R)$, which can be seen by parameterizing an $(n-1)$-dimensional cube containing $B_{P^{\prime}}\left(R^{\prime}\right)$. From now on we may assume $\operatorname{int}(S) \neq \emptyset$. Therefore we have a point $P_{1} \operatorname{in} \operatorname{int}(S)$ and a real number $r>0$ such that $B_{P_{1}}(r)$ lies in $\operatorname{int}(S)$. On the other hand, there is a point $P_{2}$ such that $S$ is in $B_{P_{2}}(R)$. The triangle inequality implies that $S$ lies in $B_{P_{1}}(2 R)$. Applying Lemma 3.1 proves the existence of a Lipschitz parameterization of the boundary. Unfortunately the Lipschitz constant $L$ has a disallowed dependence on $r$ and, moreover, the exponent on $R$ should be 1, not 2 . We can overcome these problems using John's Theorem (see [7] or [1, p. 242]). It guarantees the existence of an ellipsoid $E$, with center $P$ say, such that

$$
E \subseteq S \subseteq n(E-P)+P .
$$

After a translation by $P$ and an orthogonal transformation we can assume that $E$ is defined by $\left(x_{1} / a_{1}\right)^{2}+\cdots+\left(x_{n} / a_{n}\right)^{2}=1$ with certain real numbers $0<a_{1} \leq$ $a_{2} \leq \cdots \leq a_{n}$. Moreover, $S$ lies in a ball of radius $R$, and thus so does $E$. Hence

$$
0<a_{1} \leq a_{2} \leq \cdots \leq a_{n} \leq R .
$$

Let $\Phi$ be the endomorphism which sends $x_{i} \longrightarrow x_{i} / a_{i}$ for $1 \leq i \leq n$. Applying this map to (3.2) yields

$$
B_{0}(1) \subseteq \Phi(S) \subseteq B_{0}(n) .
$$

Applying Lemma 3.1 with $r=1$ and $R=n$ yields a map $\widetilde{\varphi}:[0,1]^{n-1} \longrightarrow \mathbb{R}^{n}$ with the image containing $\partial(\Phi(S))$ such that

$$
\left|\widetilde{\varphi}\left(\mathbf{x}_{2}\right)-\widetilde{\varphi}\left(\mathbf{x}_{1}\right)\right| \leq 8 n^{5 / 2}\left|\mathbf{x}_{2}-\mathbf{x}_{1}\right| .
$$

Since $\Phi$ is linear and bijective the maps $\Phi$ and its inverse $\Phi^{-1}$ are continuous. So the boundary of $S$ is the image under $\Phi^{-1}$ of the boundary $\partial \Phi(S)$. Thus $\Phi^{-1}(\widetilde{\varphi}(\cdot))$ is a parameterization of $\partial S$. Let us calculate a Lipschitz constant:

$$
\begin{aligned}
\left|\Phi^{-1}\left(\widetilde{\varphi}\left(\mathbf{x}_{2}\right)\right)-\Phi^{-1}\left(\widetilde{\varphi}\left(\mathbf{x}_{1}\right)\right)\right| & =\left|\Phi^{-1}\left(\widetilde{\varphi}\left(\mathbf{x}_{2}\right)-\widetilde{\varphi}\left(\mathbf{x}_{1}\right)\right)\right| \\
& \leq \sup _{|\mathbf{z}|=1}\left|\Phi^{-1}(\mathbf{z})\right|\left|\widetilde{\varphi}\left(\mathbf{x}_{2}\right)-\widetilde{\varphi}\left(\mathbf{x}_{1}\right)\right| \\
& =\sup _{|\mathbf{z}|=1}\left(\sum_{i=1}^{n}\left(a_{i} z_{i}\right)^{2}\right)^{1 / 2}\left|\widetilde{\varphi}\left(\mathbf{x}_{2}\right)-\widetilde{\varphi}\left(\mathbf{x}_{1}\right)\right| \\
& \leq \sup _{|\mathbf{z}|=1} a_{n}\left(\sum_{i=1}^{n} z_{i}^{2}\right)^{1 / 2}\left|\widetilde{\varphi}\left(\mathbf{x}_{2}\right)-\widetilde{\varphi}\left(\mathbf{x}_{1}\right)\right| \\
& =a_{n}\left|\widetilde{\varphi}\left(\mathbf{x}_{2}\right)-\widetilde{\varphi}\left(\mathbf{x}_{1}\right)\right| \\
& \leq a_{n} 8 n^{5 / 2}\left|\mathbf{x}_{2}-\mathbf{x}_{1}\right| \\
& \leq 8 n^{5 / 2} R\left|\mathbf{x}_{2}-\mathbf{x}_{1}\right| .
\end{aligned}
$$

This agrees with our claim and thereby completes the proof. 


\section{Proof of Theorem 2.8}

Let $\Gamma_{0}, \Gamma_{1}$ be the paths of two simple, piecewise smooth curves of arc lengths $\left|\Gamma_{0}\right|,\left|\Gamma_{1}\right|$ (as the curves are simple, the arc lengths depend only on the paths). Poincaré's formula tells us (see [11, p. 111, eq. (7.11))

$$
\int_{\mathfrak{M}} \mathfrak{n} \mathrm{d} K=4\left|\Gamma_{0}\right|\left|\Gamma_{1}\right|
$$

where $\mathfrak{M}$ is the group of motions $\phi$ in the plane, $\mathfrak{n}=\mathfrak{n}(\phi)=\left|\Gamma_{1} \cap \phi\left(\Gamma_{0}\right)\right|$ (possibly infinite) is the intersection number of $\Gamma_{1}$ and $\phi\left(\Gamma_{0}\right)$, and $\mathrm{d} K$ is the kinematic density. Maak [8] gave a generalization of formula (4.1), but Maak's definition of the intersection number $\mathfrak{n}$ is different from ours, so that we prefer not to rely on his result. Now $\partial S$ lies in a ball of radius $R$, say $B_{P}(R)$. Suppose $\Gamma_{0}$ is a line segment. Let $\mathfrak{M}_{1}=\mathfrak{M}_{1}\left(\Gamma_{0}\right)$ be the subset of $\mathfrak{M}$ defined by $\mathfrak{n}(\phi)=\left|\partial S \cap \phi\left(\Gamma_{0}\right)\right| \leq 2 s$. Then condition $(I I)$ of Definition 2.7 above implies that

$$
\int_{\mathfrak{M}} \mathfrak{n} \mathrm{d} K=\int_{\mathfrak{M}_{1}} \mathfrak{n} \mathrm{d} K
$$

Moreover, $\mathfrak{n}(\phi)=0$ for any $\phi$ with $\phi\left(\Gamma_{0}\right) \cap B_{P}(R)=\emptyset$. The measure of the set of motions $\phi$ with $\phi\left(\Gamma_{0}\right) \cap B_{P}(R) \neq \emptyset$ is not hard to compute (see [11], p. 90, (6.33)), and one finds

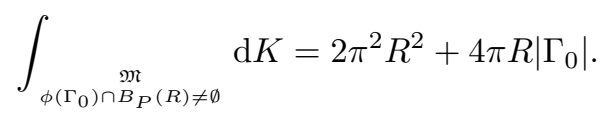

Now combining (4.1), (4.2) and (4.3) yields

$$
\begin{aligned}
& 4\left|\Gamma_{0}\right||\partial S|=\int_{\substack{\left(\Gamma_{0}\right) \cap B_{P}(R) \neq \emptyset \\
\mathfrak{m}_{1}}} \mathfrak{n d} K \leq 2 s \int_{\substack{\left.\Gamma_{0}\right) \cap M_{P}(R) \neq \emptyset \\
\mathfrak{M}_{1}}} \mathrm{~d} K=2 s \int_{\substack{\left.\Gamma_{0}\right) \cap B_{P}(R) \neq \emptyset \\
\mathfrak{m}}} \mathrm{d} K \\
& =2 s\left(2 \pi^{2} R^{2}+4 \pi R\left|\Gamma_{0}\right|\right) \text {. }
\end{aligned}
$$

Thus we have $|\partial S| \leq 2 \pi s R+\pi^{2} R^{2} s /\left|\Gamma_{0}\right|$ for any length $\left|\Gamma_{0}\right|$, and therefore $|\partial S| \leq$ $2 \pi s R$. It is well known that the path of a rectifiable curve can be parameterized by the arc length. Let $\psi$ be such a parameterization of $\partial S$, scaled from $[0,|\partial S|]$ to $[0,1]$. Then we have $\left|\psi(t)-\psi\left(t^{\prime}\right)\right| \leq|\partial S|\left|t-t^{\prime}\right|$. This shows that $\partial S$ lies in $\operatorname{Lip}(2,1,2 \pi s R)$ and thereby completes the proof.

\section{Proof of Proposition 2.9}

By definition $\mathcal{A}_{S \cap \Lambda}$ is the minimal affine subspace containing $S \cap \Lambda$. For brevity let us write $\mathcal{A}$ for $\mathcal{A}_{S \cap \Lambda}$. Now we have $\mathcal{A}=\operatorname{span}\{v-w: v \in S \cap \Lambda\}+w$ for any $w$ in $\mathcal{A}$. In particular we can assume $w \in S \cap \Lambda$. Thus we can find a basis $v_{1}-w, \ldots, v_{l}-w$ of $W=\operatorname{span}\{v-w: v \in S \cap \Lambda\}$ with $w, v_{1}, \ldots, v_{l} \in S \cap \Lambda$. Now clearly $(S \cap \Lambda) \subseteq \mathcal{A}=W+w$. Hence we conclude

$$
\begin{aligned}
|S \cap \Lambda|=|(S \cap(W+w)) \cap(\Lambda \cap(W+w))| & =|((S-w) \cap W) \cap(\Lambda \cap W)| \\
& =\left|S^{\prime} \cap \Lambda^{\prime}\right|,
\end{aligned}
$$

where $S^{\prime}=(S-w) \cap W$ and $\Lambda^{\prime}=\Lambda \cap W$. Of course $S^{\prime}$ is convex and lies in some $\mathbb{R}^{l}$. First suppose $l=1$. Then, as $|S \cap \Lambda|>1$, we have $\operatorname{Vol}_{1}\left(S^{\prime}\right) \geq \lambda_{1}\left(\Lambda^{\prime}\right) \geq \lambda_{1}$ and hence

$$
|S \cap \Lambda| \leq\left(\operatorname{Vol}_{1}\left(S^{\prime}\right)\right) / \lambda_{1}+1 \leq c_{3}(1)\left(\operatorname{Vol}_{1}\left(S^{\prime}\right)\right) / \lambda_{1} .
$$


From now on we assume $l>1$. Suppose $S^{\prime}$ (as a subset of $\mathbb{R}^{l}$ ) has empty interior. Then $S^{\prime}$ lies in an affine subspace of dimension $l-1$, and so does $(S \cap \Lambda) \subseteq S^{\prime}+w$. But this contradicts the minimality of $\mathcal{A}$. Therefore we can apply John's Theorem; i.e., we can find an ellipsoid $E$, with center $P \in S^{\prime}$, say, such that

$$
E \subseteq S^{\prime} \subseteq l(E-P)+P
$$

There exists a translation $t_{P}$ by $P$ and an orthogonal map $\psi$ such that $\psi \circ t_{P}(E)$ is defined by $\left(x_{1} / a_{1}\right)^{2}+\cdots+\left(x_{l} / a_{l}\right)^{2}=1$ for certain positive reals $a_{1}, \ldots, a_{l}$. Put $R=\left(a_{1} \cdots a_{l}\right)^{1 / l}$. Moreover, there exists a linear endomorphism $\Phi$ with $\operatorname{det} \Phi=1$ that sends $E-P$ to $B_{0}(R)$. Hence we have $\Phi(E)=B_{P^{\prime}}(R)$, with $P^{\prime}=\Phi(P)$, and therefore

$$
B_{P^{\prime}}(R) \subseteq \Phi\left(S^{\prime}\right) \subseteq B_{P^{\prime}}(l R)
$$

Note that $0=w-w \in S^{\prime}$. Using (5.1) and the triangle inequality yields $\Phi\left(S^{\prime}\right) \subseteq$ $B_{0}(2 l R)$. The linearly independent lattice points $v_{1}-w, \ldots, v_{l}-w$ all lie in $S^{\prime}$ and thus

$$
\lambda_{l}^{\prime} \leq \max \left\{\left|\Phi\left(v_{1}-w\right)\right|, \ldots,\left|\Phi\left(v_{l}-w\right)\right|\right\} \leq 2 l R
$$

for the successive minimum $\lambda_{l}^{\prime}$ of the lattice $\Phi\left(\Lambda^{\prime}\right)$. Applying Theorem 2.3 with $\Phi\left(S^{\prime}\right), \Phi\left(\Lambda^{\prime}\right)$ and using $2 l R \geq \lambda_{l}^{\prime}$, we conclude

$$
\begin{aligned}
\left|S^{\prime} \cap \Lambda^{\prime}\right|=\left|\Phi\left(S^{\prime}\right) \cap \Phi\left(\Lambda^{\prime}\right)\right| & \leq\left|B_{P^{\prime}}(l R) \cap \Phi\left(\Lambda^{\prime}\right)\right| \\
& \leq \frac{\operatorname{Vol}\left(B_{P^{\prime}}(l R)\right)}{\operatorname{det} \Phi\left(\Lambda^{\prime}\right)}+c_{1}(l, 1) \frac{(2 l R)^{l-1}}{\lambda_{1}^{\prime} \cdots \lambda_{l-1}^{\prime}} \frac{2 l R}{\lambda_{l}^{\prime}} .
\end{aligned}
$$

However, due to the lack of a published reference, we will apply Theorem 2.6 instead. From Lemma 3.1 we see that $\partial B_{P^{\prime}}(l R)$ lies in $\operatorname{Lip}(l, 1,8 \sqrt{l-1} l R)$, which gives

$$
\left|S^{\prime} \cap \Lambda^{\prime}\right| \leq \frac{\operatorname{Vol}\left(B_{P^{\prime}}(l R)\right)}{\operatorname{det} \Phi\left(\Lambda^{\prime}\right)}+c_{2}(l) \frac{(8 \sqrt{l-1} l R)^{l}}{\lambda_{1}^{\prime} \cdots \lambda_{l}^{\prime}} .
$$

Next we observe

$$
\begin{aligned}
\operatorname{Vol}\left(B_{P^{\prime}}(R)\right) & \leq \operatorname{Vol}\left(\Phi\left(S^{\prime}\right)\right) \leq \operatorname{Vol}\left(B_{P^{\prime}}(l R)\right) \\
\operatorname{Vol}\left(\Phi\left(S^{\prime}\right)\right) & =\operatorname{Vol}\left(S^{\prime}\right)=\operatorname{Vol}_{l}(S \cap \mathcal{A})
\end{aligned}
$$

Writing $c_{2}^{\prime}=1+c_{2}(l)(4 \sqrt{l-1})^{l} l$ ! and applying Minkowski's Second Theorem, we conclude

$$
\left|S^{\prime} \cap \Lambda^{\prime}\right| \leq c_{2}^{\prime} \frac{\operatorname{Vol}\left(B_{P^{\prime}}(l R)\right)}{\operatorname{det} \Phi\left(\Lambda^{\prime}\right)}=c_{2}^{\prime} l^{l} \frac{\operatorname{Vol}\left(B_{P^{\prime}}(R)\right)}{\operatorname{det} \Lambda^{\prime}} \leq c_{2}^{\prime} l^{l} \frac{\operatorname{Vol}_{l}(S \cap \mathcal{A})}{\operatorname{det} \Lambda^{\prime}} .
$$

Finally, we use Minkowski's Second Theorem once more, together with

$$
\lambda_{1}\left(\Lambda^{\prime}\right) \cdots \lambda_{l}\left(\Lambda^{\prime}\right) \geq \lambda_{1} \cdots \lambda_{l}
$$

and the result drops out after noting that $(2 l)^{l} c_{2}^{\prime} / \operatorname{Vol}\left(B_{0}(1)\right) \leq 8^{l} l^{3 l(l / 2+1)}$. 


\section{Proof of Corollary 2.11}

For a vector $\mathbf{y}=\left(y_{0}, y_{1}, \ldots, y_{n-1}\right) \in \mathbb{Z}^{n}$ we write $W_{\mathbf{y}}$ for the orthogonal complement of $\mathbf{y} \mathbb{R}$ in $\mathbb{R}^{n}$. Put $\Lambda_{\mathbf{y}}=W_{\mathbf{y}} \cap \mathbb{Z}^{n}$ and $\mathbf{y}^{d}=\left(y_{0}^{d}, y_{1}^{d}, \ldots, y_{n-1}^{d}\right)$. If $\mathbf{y}$ is primitive (i.e., $\mathbf{y} \neq \mathbf{0}$ and $\operatorname{gcd}\left(y_{0}, y_{1}, \ldots, y_{n-1}\right)=1$ ), then $\Lambda_{\mathbf{y}}$ is a lattice of rank $n-1$ with $\operatorname{det} \Lambda_{\mathbf{y}}=|\mathbf{y}|$. Let $\Lambda_{\mathbf{y}}^{*}$ be the set of primitive vectors in $\Lambda_{\mathbf{y}}$, and set $|\mathbf{y}|_{\infty}=\max \left\{\left|y_{0}\right|,\left|y_{1}\right|, \ldots,\left|y_{n-1}\right|\right\}$.

We fix a primitive vector $\mathbf{y} \in \mathbb{Z}^{3}$ and we count all primitive vectors $\mathbf{x}$ satisfying (2.2) and $|\mathbf{x}|_{\infty}=H\left(\left(x_{0}: x_{1}: x_{2}\right)\right) \leq t / H\left(\left(y_{0}: y_{1}: y_{2}\right)\right)=t /|\mathbf{y}|_{\infty}$. Then summing over all primitive vectors $\mathbf{y}$ we see that

$$
N\left(V_{d}, t\right) \leq \sum_{\substack{\mathbf{y} \in \mathbb{Z}^{3} \\ \text { primitive }}}\left|\left[-\frac{t}{|\mathbf{y}|_{\infty}}, \frac{t}{|\mathbf{y}|_{\infty}}\right]^{3} \cap \Lambda_{\mathbf{y}^{d}}^{*}\right|
$$

Let us first estimate the number of $(\mathbf{x}, \mathbf{y})$ with $x_{0} x_{1} x_{2}=0$. By symmetry we can assume $x_{2}=0$. Then the term in our sum above becomes

$$
\left|\left[-\frac{t}{|\mathbf{y}|_{\infty}}, \frac{t}{|\mathbf{y}|_{\infty}}\right]^{2} \cap \Lambda_{\left(y_{0}^{d}, y_{1}^{d}\right)}^{*}\right| .
$$

We write $\left(y_{0}, y_{1}\right)=m\left(y_{0}^{\prime}, y_{1}^{\prime}\right)=m \mathbf{y}^{\prime}$ with coprime $y_{0}^{\prime}, y_{1}^{\prime}$. Note that if $y_{0}=y_{1}=0$, then $y_{2}= \pm 1$ and so we have $\ll t^{2}$ of these points $(\mathbf{x}, \mathbf{y})$. Thus we can assume $m \mathbf{y}^{\prime} \neq 0$. Now $\Lambda_{\left(y_{0}^{d}, y_{1}^{d}\right)}=\Lambda_{\mathbf{y}^{\prime d}}$ is a 1-dimensional lattice with determinant $\left|\mathbf{y}^{\prime d}\right|$. This means that any primitive vector $\left(x_{0}, x_{1}\right)$ in $\Lambda_{\mathbf{y}^{\prime d}}$ has length $\left|\mathbf{y}^{\prime d}\right|$ and, moreover, there are only 2 primitive vectors in each $\Lambda_{\mathbf{y}^{\prime d}}$. So (6.2) is either 0 or 2 , and if it is 2, then: $\sqrt{2} t /|\mathbf{y}|_{\infty} \geq\left|\left(x_{0}, x_{1}\right)\right|=\left|\mathbf{y}^{\prime d}\right| \geq\left|\mathbf{y}^{\prime d}\right|_{\infty}$. As $|\mathbf{y}|_{\infty} \geq m\left|\mathbf{y}^{\prime}\right|_{\infty}$ we conclude $\left|\mathbf{y}^{\prime}\right|_{\infty} \leq(\sqrt{2} t / m)^{1 /(d+1)}$. This shows that the total number of points $(\mathbf{x}, \mathbf{y})$ with $x_{0} x_{1} x_{2}=0$ and $m \mathbf{y}^{\prime} \neq 0$ is

$$
\leq 3 \sum_{m=1}^{[t]} \sum_{\substack{\mathbf{y}=\left(m y^{\prime}, m y_{1}^{\prime}, y_{2}\right) \\\left|y_{2} \leq t\\\right| y_{0}^{\prime}|,| y_{1}^{\prime} \mid \leq(\sqrt{2} t / m)^{1 /(d+1)}}}\left|\left[-\frac{t}{|\mathbf{y}|_{\infty}}, \frac{t}{|\mathbf{y}|_{\infty}}\right]^{2} \cap \Lambda_{\mathbf{y}^{\prime d}}^{*}\right| \leq 6 \sum_{m=1}^{[t]} \sum_{\substack{\mathbf{y}=\left(m y^{\prime}, m y_{1}^{\prime}, y_{2}\right) \\\left|y_{2} \leq t\\\right| y_{0}^{\prime}|,| y_{1}^{\prime} \mid \leq(\sqrt{2} t / m)^{1 /(d+1)}}}
$$

The latter is

$$
\ll \sum_{m=1}^{[t]} t(t / m)^{2 /(d+1)}=t^{1+2 /(d+1)} \sum_{m=1}^{[t]} m^{-2 /(d+1)} \ll \begin{cases}t^{2} \log (2 t): & \text { if } d=1, \\ t^{2}: & \text { if } d>1 .\end{cases}
$$

It remains to estimate the number of points $(\mathbf{x}, \mathbf{y})$ that satisfy $x_{0} x_{1} x_{2} \neq 0$. We note that for fixed $x_{0}, x_{1}, x_{2}$ with $x_{0} x_{1} x_{2} \neq 0$ the equation $x_{0} y_{0}^{d}+x_{1} y_{1}^{d}+x_{2} y_{2}^{d}=0$ defines an affine irreducible variety over $\mathbb{R}$ of dimension 2 and degree $d$. To see this it suffices to show the irreducibility of $f(x)=x^{d}+y^{d}-1$ in $\mathbb{C}[y][x]$. But the latter follows from Eisenstein's criterion. Next we need an upper bound for the term in the sum of (6.1). Now either $\left[-t /|\mathbf{y}|_{\infty}, t /|\mathbf{y}|_{\infty}\right]^{3} \cap \Lambda_{\mathbf{y}^{d}}^{*}$ is not contained in a line or all points lie on one single line (passing through the origin). In the former case we can apply Corollary 2.10 to deduce the upper bound $\ll t^{2}|\mathbf{y}|^{-(d+2)}$, and in the latter we have at most 2 primitive vectors $\mathbf{x}$. Hence the number of points $(\mathbf{x}, \mathbf{y})$ that satisfy $x_{0} x_{1} x_{2} \neq 0$ is

$$
\ll \sum_{\substack{\mathbf{y} \text { primitive } \\|\mathbf{y}| \infty \leq t}} \frac{t^{2}}{|\mathbf{y}|^{d+2}}+\sum_{\substack{\mathbf{y} \text { primitive } \\|\mathbf{y}| \infty \leq t}} 1^{*}(t, \mathbf{y})
$$


with

$$
1^{*}(t, \mathbf{y})= \begin{cases}1: & \text { if } \lambda_{1}\left(\Lambda_{\mathbf{y}^{d}}\right)|\mathbf{y}|_{\infty} \leq \sqrt{3} t \\ 0: & \text { otherwise }\end{cases}
$$

The simple calculation

$$
\sum_{\substack{\mathbf{y} \\ \text { primitive } \\|\mathbf{y}| \infty \leq t \leq t}} \frac{t^{2}}{|\mathbf{y}|^{d+2}} \leq \sum_{e=1}^{[t]} t^{2} \frac{\left|\left\{\mathbf{y}:|\mathbf{y}|_{\infty}=e\right\}\right|}{e^{d+2}} \ll t^{2} \sum_{e=1}^{[t]} \frac{e^{2}}{e^{d+2}}=t^{2} \sum_{e=1}^{[t]} e^{-d}
$$

shows that the first sum in (6.3) is bounded from above by the right-hand side of (2.3). To estimate the second sum we need an upper bound on the number of primitive vectors $\mathbf{y}$ with $\lambda_{1}\left(\Lambda_{\mathbf{y}^{d}}\right)|\mathbf{y}|_{\infty} \ll t$. We distinguish two cases. For brevity we write $\tau=\tau(t)=t^{\left(3 d^{2}-4 d+2\right) /(5 d-1)}, \lambda_{1}$ for $\lambda_{1}\left(\Lambda_{\mathbf{y}^{d}}\right)$ and $\lambda_{2}$ for $\lambda_{2}\left(\Lambda_{\mathbf{y}^{d}}\right)$.

Case $1\left(\lambda_{2} / \lambda_{1} \leq \tau\right)$. Here we find

$$
|\mathbf{y}| \ll\left(\operatorname{det} \Lambda_{\mathbf{y}^{d}}\right)^{1 / d} \ll\left(\lambda_{1} \lambda_{2}\right)^{1 / d} \leq\left(\lambda_{1} \lambda_{1} \tau\right)^{1 / d}=\lambda_{1}^{2 / d} \tau^{1 / d} .
$$

As we have $\lambda_{1}|\mathbf{y}| \ll \lambda_{1}|\mathbf{y}|_{\infty} \ll t$ we conclude $|\mathbf{y}| \ll t^{2 /(d+2)} \tau^{1 /(d+2)}$. This means that we have at most

$$
\ll t^{6 /(d+2)} \tau^{3 /(d+2)}=t^{6 /(d+2)+3\left(3 d^{2}-4 d+2\right) /((d+2)(5 d-1))}=t^{9 d /(5 d-1)}
$$

possibilities for $\mathbf{y}$ in case 1 , and this in turn proves Corollary 2.11 in case 1 .

Case $2\left(\lambda_{2} / \lambda_{1}>\tau\right)$. Here we have

$$
t \gg \lambda_{1}|\mathbf{y}| \gg \lambda_{1}\left(\lambda_{1} \lambda_{2}\right)^{1 / d}>\lambda_{1}\left(\lambda_{1}^{2} \tau\right)^{1 / d}=\lambda_{1}^{(d+2) / d} \tau^{1 / d},
$$

and therefore $\lambda_{1} \ll t^{d /(d+2)} \tau^{-1 /(d+2)}$. Now let $x=\left(x_{0}, x_{1}, x_{2}\right)$ be a primitive vector in $\Lambda_{\mathbf{y}^{d}}$ with $|\mathbf{x}|=\lambda_{1}$ and $x_{0} x_{1} x_{2} \neq 0$. Thus we have $|\mathbf{x}| \ll t^{d /(d+2)} \tau^{-1 /(d+2)}$, and the height bound gives $|\mathbf{x}||\mathbf{y}| \ll t$. Now for each fixed vector $\mathbf{x}$ as above we count the number of primitive vectors $\mathbf{y}$ such that $\mathbf{x}$ is a minimal vector of $\Lambda_{\mathbf{y}^{d}}$ and $|\mathbf{x}||\mathbf{y}| \ll t$. Then we sum these upper bounds over all primitive vectors $\mathbf{x}$ with $|\mathbf{x}| \ll t^{d /(d+2)} \tau^{-1 /(d+2)}$ and $x_{0} x_{1} x_{2} \neq 0$. So the number of vectors $\mathbf{y}$ is

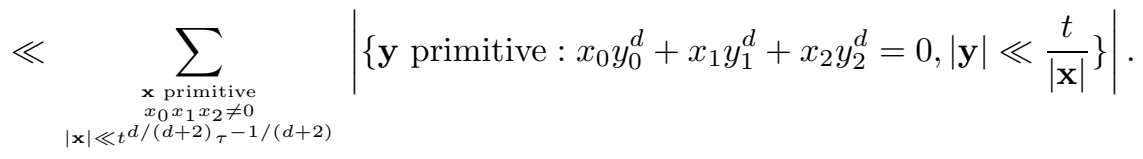

The term in the sum above is certainly $\ll t^{2} /|\mathbf{x}|^{2}$. Similarly as in (6.4) this yields the upper bound $\ll t^{2+d /(d+2)} \tau^{-1 /(d+2)}$ for the number of vectors $\mathbf{y}$, and this in turn proves Corollary 2.11 for $d=1$. However, for $d>1$ we apply a general result of Pila (Theorem A in [10]) to deduce the better bound $\ll_{\epsilon}(t /|\mathbf{x}|)^{1+1 / d+\epsilon}$ for the term in the sum above (here we used the fact that for fixed $x_{0}, x_{1}, x_{2}$ with $x_{0} x_{1} x_{2} \neq 0$ the equation $x_{0} y_{0}^{d}+x_{1} y_{1}^{d}+x_{2} y_{2}^{d}=0$ defines an affine irreducible variety over $\mathbb{R}$ of dimension 2 and degree $d$ ). This gives the upper bound

$$
\ll_{\epsilon} t^{1+2\left(d^{2}+1\right) /(d(d+2))+\epsilon} \tau^{-(2 d-1) /(d(d+2))}=t^{9 d /(5 d-1)+\epsilon}
$$

for the number of vectors $\mathbf{y}$ and thereby completes the proof of Corollary 2.11, 


\section{ACKNOWLEDGEMENTS}

The author thanks David Masser for interesting and significant comments that simplified the original proof of Lemma 3.1, and Christopher Frei for helpful remarks on a previous version of this manuscript. Moreover, he thanks the referee for many detailed and valuable remarks.

\section{REFERENCES}

1. K. Ball, Ellipsoids of maximal volume in convex bodies, Geom. Dedicata 41 (1992), no. 2, 241-250. MR.1153987 (93k:52006)

2. H. F. Blichfeldt, The April meeting of the San Francisco section of the AMS, The American Math. Monthly 28, no. 6/7 (1920/21), 285-292.

3. M. R. Bridson and A. Haefliger, Metric Spaces of Non-Positive Curvature, Springer, 1999. MR.1744486 (2000k:53038)

4. H. Davenport, On a principle of Lipschitz, J. London Math. Soc. 26 (1951), 179-183. MR0043821(13:323d)

5. X. Gao, On Northcott's Theorem, Ph.D. Thesis, University of Colorado (1995).

6. M. Henk and J. M. Wills, A Blichfeldt-type inequality for the surface area, Monatsh. Math. 154 (2008), 135-144. MR2419059 (2009c:52026)

7. F. John, Extremum problems with inequalities as subsidiary conditions, Studies and Essays Presented to R. Courant on His 60th Birthday, Interscience Publishers, Inc., New York, NY, 1948, pp. 187-204. MR0030135(10:719b)

8. W. Maak, Schnittpunktanzahl rektifizierbarer und nichtrektifizierbarer Kurven, Math. Ann. 118 (1942), 299-304. MR0008463 (5:10d)

9. D. W. Masser and J. D. Vaaler, Counting algebraic numbers with large height. II, Trans. Amer. Math. Soc. 359 (2007), 427-445. MR2247898 (2008m:11208)

10. J. Pila, Density of integral and rational points on varieties, Astérisque 228 (1995), 183-187. MR:1330933 (96b:11043)

11. L. A. Santaló, Integral Geometry and Geometric Probability, Addison Wesley, 1976. MR 0433364 (55:6340)

12. W. M. Schmidt, Northcott's theorem on heights. II. The quadratic case, Acta Arith. 70 (1995), 343-375. MR1330740 (96a:11059)

13. M. Widmer, Counting points of fixed degree and bounded height, Acta Arith. 140, no. 2 (2009), 145-168. MR2558450 (2010j:11109)

14. _ Counting points of fixed degree and bounded height on linear varieties, J. Number Theory 130 (2010), 1763-1784. MR2651154

15. _ Counting primitive points of bounded height, Trans. Amer. Math. Soc. 362 (2010), 4793-4829. MR2645051

16. $ـ$ On number fields with nontrivial subfields, to appear in Int. J. Number Theory (2010).

17. I. M. Yaglom and V. G. Boltjanskiur, Convex figures (translated by P. J. Kelly and L. F. Walton), Holt, Rinehart and Winston, New York, 1960. MR0123962 (23:A1283)

Department of Mathematics, Technische Universität Graz, Steyrergasse 30/II, 8010 Graz, Austria

E-mail address: widmer@math.tugraz.at 